\title{
Two pressure-induced structural phase transitions in TiOCl
}

\author{
J. Ebad-Allah, ${ }^{1}$ A. Schönleber, ${ }^{2}$ S. van Smaalen, ${ }^{2}$ M. Hanfland, ${ }^{3}$ M. Klemm, ${ }^{1}$ \\ S. Horn, ${ }^{1}$ S. Glawion, ${ }^{4}$ M. Sing, ${ }^{4}$ R. Claessen ${ }^{4}$, and C. A. Kuntscher ${ }^{1 *}$ \\ 1 Experimentalphysik 2, Universität Augsburg, D-86135 Augsburg, Germany \\ 2 Laboratory of Crystallography, Universität Bayreuth, D-95440 Bayreuth, Germany \\ 3 European Synchrotron Radiation Facility, BP 220, F-38043 Grenoble, France \\ 4 Experimentelle Physik 4, Universität Würzburg, D-97074 Würzburg, Germany
}

(Dated: May 27, 2022)

\begin{abstract}
We studied the crystal structure of $\mathrm{TiOCl}$ up to pressures of $p=25 \mathrm{GPa}$ at room temperature by x-ray powder diffraction measurements. Two pressure-induced structural phase transitions are observed: At $p_{c 1} \approx 15 \mathrm{GPa}$ emerges an $2 a \times 2 b \times c$ superstructure with $b$-axis unique monoclinic symmetry (space group $\mathrm{P} 22_{1} / m$ ). At $p_{c 2} \approx 22 \mathrm{GPa}$ all lattice parameters of the monoclinic phase show a pronounced anomaly. A fraction of the sample persists in the ambient orthorhombic phase (space group Pmmn) over the whole pressure range.

PACS numbers: $62.50 .-\mathrm{p}, 61.50 . \mathrm{Ks}$
\end{abstract}

\section{INTRODUCTION}

Recently, high-pressure studies on the low-dimensional Mott-Hubbard insulators $\mathrm{TiOCl}$ and $\mathrm{TiOBr}$ have demonstrated the high sensitivity of their electronic and structural properties regarding the application of pressure. The first high-pressure experiments on $\mathrm{TiOCl}$ and $\mathrm{TiOBr}$ single crystals at room temperature revealed strong changes in the optical response in the infrared frequency range, with the suppression of the transmittance and increasing optical conductivity at high pressures $\frac{1-3}{\underline{3}}$ Based on these results the possibility of a pressure-induced insulator-to-metal transition was suggested. However, subsequent electrical transport measurements on powder samples did not confirm a metallization of $\mathrm{TiOCl}$ at high pressure and found only an anomaly in the pressureinduced decrease of the charge gap at $\approx 15 \mathrm{GPa} .4$ A controversy regarding the high-pressure electronic properties of $\mathrm{TiOCl}$ also exists from the theoretical point of view: $A b$ initio calculations were carried out based on density functional theory within the $\mathrm{LDA}+\mathrm{U}$ approximation ${ }^{5}$ and using Car-Parrinello molecular dynamics. $\underline{6}$ While the former claims an insulating phase up to at least $30 \mathrm{GPa}$, the latter predicts the metallic character of the highpressure phases.

According to both experiment and theory, TiOCl exhibits structural phase transitions under pressure at room temperature. Signatures of a structural phase transition in $\mathrm{TiOCl}$ and $\mathrm{TiOBr}$ under pressure were first observed in x-ray powder diffraction studies ${ }_{2.7}^{2.7}$ This structural phase transition coincides with an anomaly in the pressure-dependent electrical and optical properties at $p_{c 1} \approx 15 \mathrm{GPa}: \stackrel{2,4,7}{ }$ Blanco-Canosa et al $\stackrel{5}{\frac{5}{*}}$ confirmed the occurrence of a structural phase transition, but the corresponding critical pressure was considerably lower $(\approx 10 \mathrm{GPa})$. The high-pressure phase was specified as $b$-axis unique monoclinic $P 2_{1} / m$ phase with a strong dimerization of the $1 \mathrm{D}$ spin chain along $b{ }^{\frac{5}{5}}$ It was furthermore claimed ${ }^{5}$ that the dimerization is of pure electronic origin, i.e., resembles that of a conventional Peierls insulator, and that the magnetic interaction plays only a minor role at high pressure. It is noticed here that the reported symmetry ${ }^{\underline{5}}$ is not a subgroup of the ambient Pmmn symmetry. The high-pressure phase thus is not a simple superstructure of the structure at ambient conditions, but it requires major rearrangements affecting the connectivity between atoms. These might be provided by the observed monoclinic angle of $\beta \sim 99^{\circ}$ as compared to $90^{\circ}$ in the orthorhombic phase. The reported atomic positions at $2 e:\left(\frac{1}{4}, y, z\right)$ would require a mirror plane perpendicular to the $a$-axis and thus are incompatible with the reported monoclinic $b$-axis $\frac{\underline{5}}{}$ Besides, several aspects of the high-pressure phases still need to be clarified, e.g., the values of the critical pressures and possible superstructures being induced at higher pressures.

Here we present x-ray diffraction data on $\mathrm{TiOCl}$ for an extended pressure range up to $\sim 25 \mathrm{GPa}$. Our goal is to clarify the inconsistencies regarding the experimental findings for the high-pressure crystal structure of TiOCl. Besides a transition from the orthorhombic Pmmn to the monoclinic $P 2_{1} / m$ crystal structure with an $2 a \times 2 b \times c$ superstructure, we find a pressure-induced isostructural phase transition for the monoclinic phase with anomalies in the lattice parameters.

\section{EXPERIMENT}

Single crystals of $\mathrm{TiOCl}$ were synthesized by gas transport from $\mathrm{TiCl}_{3}$ and $\mathrm{TiO}_{2} \stackrel{10}{\underline{10}} \mathrm{TiOCl}$ crystallizes in the space group Pmmn at ambient conditions and consists of distorted $\mathrm{TiO}_{4} \mathrm{Cl}_{2}$ octahedra. Pressure-dependent x-ray powder diffraction measurements at room temperature were carried out with monochromatic radiation $(\lambda=0.4128 \AA)$ at beamline ID09A of the European Synchrotron Radiation Facility (ESRF) at Grenoble. Crystals were gently ground and placed into a diamond anvil cell (DAC). The material of the gasket was stainless steel, and its initial thickness and hole diameter was $40 \mu m$ and $150 \mu m$, respectively. The ap- 


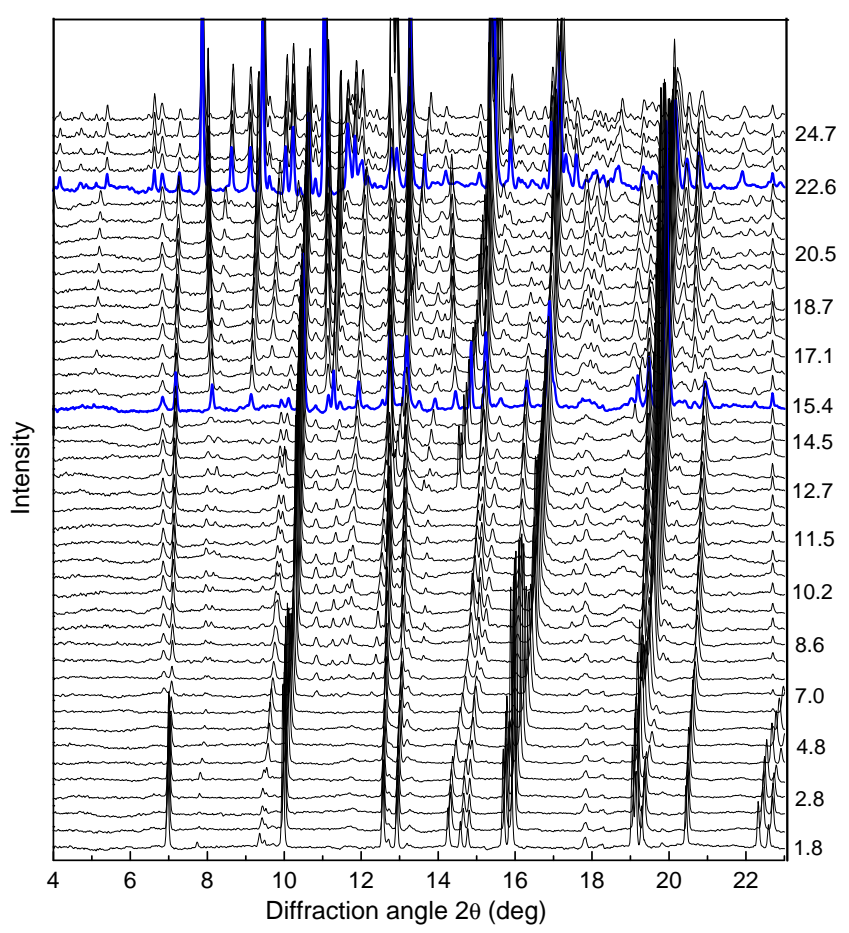

FIG. 1: Room-temperature x-ray powder diffraction diagrams of $\mathrm{TiOCl}$ at high pressures. The numbers on the right, vertical axis indicate the applied pressures in GPa. The diffraction diagrams at the critical pressures $\mathrm{p}_{c 1}=15.4 \mathrm{GPa}$ and $\mathrm{p}_{c 2}=22.6 \mathrm{GPa}$ are highlighted by bold, blue lines.

plied pressures $p$ were determined with the ruby fluorescence method $\stackrel{12}{\underline{1}}$ Helium served as hydrostatic pressuretransmitting medium. Diffraction patterns were recorded with an image plate detector and then integrated with FIT2 $\mathrm{D}^{13}$ to yield intensity vs $2 \theta$ diagrams. The DAC was rotated by $\pm 3^{\circ}$ during the exposure to improve the powder averaging. We carried out LeBail fits of the diffraction data using the Jana2006 software, 14 in order to determine the lattice parameters as a function of pressure. Rietveld refinements of the diffraction data could not be carried out due to the preferred orientation of the crystallites inside the DAC, as described earlier $\stackrel{\underline{7}}{?}$

\section{RESULTS AND DISCUSSION}

Fig. 1 1 shows the diffraction diagrams of $\mathrm{TiOCl}$ for all measured pressures. Fundamental changes in the diffraction diagrams are observed at 15.4 and $22.6 \mathrm{GPa}$, which define the critical pressures $\mathrm{p}_{c 1}$ and $\mathrm{p}_{c 2}$ for the two structural phase transitions. The x-ray powder diffraction diagrams of $\mathrm{TiOCl}$ for selected pressures up to $\sim 25 \mathrm{GPa}$ are presented in Fig. 2 Up to the critical pressure $p_{c 1} \approx 15 \mathrm{GPa}$ the diffraction diagrams can be described by LeBail fits applying symmetry and lattice parameters of the ambient-pressure, orthorhombic crystal structure (space group Pmmn) and assuming contributions from

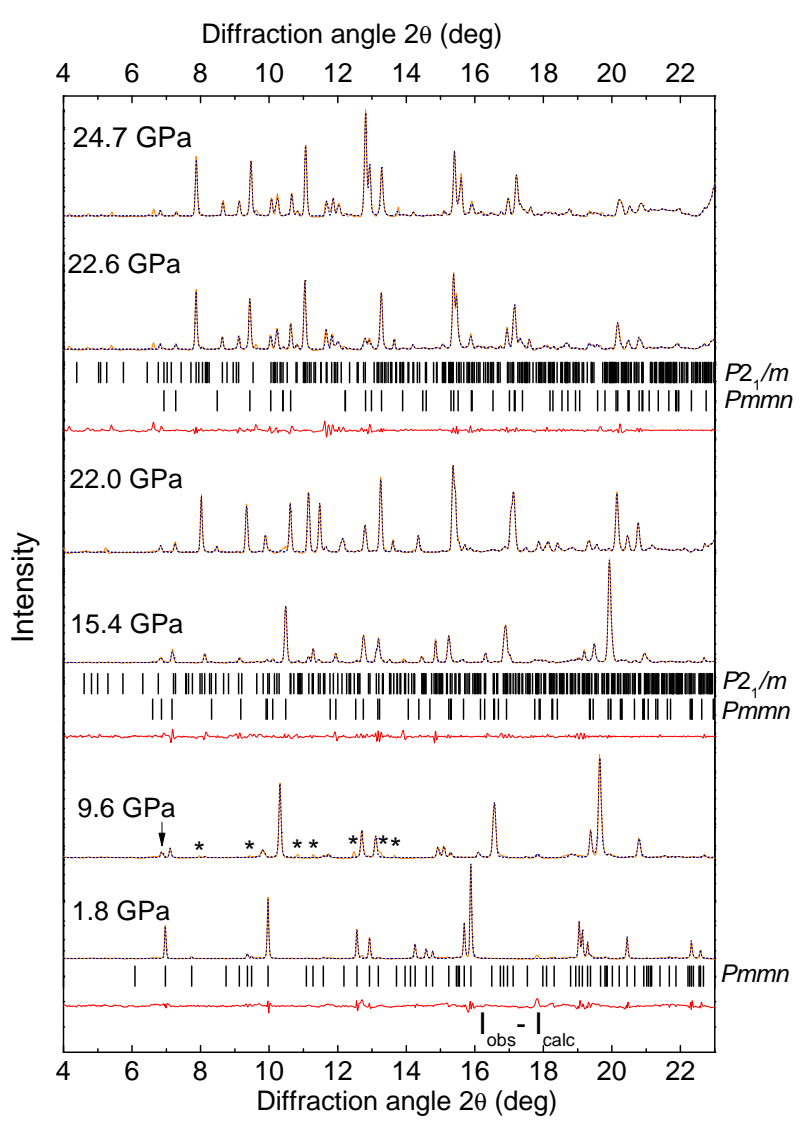

FIG. 2: Room-temperature x-ray powder diffraction diagrams (full, orange lines) of $\mathrm{TiOCl}$ at high pressures together with the LeBail fits (dotted, blue lines). For the pressures 1.8, 15.4 , and $22.6 \mathrm{GPa})$ the difference curve $\left(I_{\text {obs }}-I_{\text {calc }}\right)$ between the diffraction diagram and the LeBail fit is shown. Markers show the calculated peak positions for the various phases. The arrow indicates the reflection from the ruby chip. The asterisks mark the reflections due to the monoclinic phase already present above $\sim 7 \mathrm{GPa}$.

the ruby chips added for the pressure determination. The lattice parameters and unit cell volume of the orthorhombic crystal structure obtained by the LeBail fits are shown in Fig. 3 and are in agreement with those reported in Refs. [7,8]. We find a slightly nonlinear decrease of the lattice parameters with increasing pressure.

Above $P_{c 1} \approx 15 \mathrm{GPa}$ the diffraction diagrams can no longer be described by a single phase, but a good fit of the data can be achieved by assuming the coexistence of two phases - namely an orthorhombic phase (space group $P m m n$ ) and a monoclinic phase (space group $P 2_{1} / m$, $b$-axis unique) with double $a$ - and $b$-axes. The lattice parameters and the angle $\beta$ of the monoclinic unit cell hardly change up to $\sim 22 \mathrm{GPa}$ (see Figs. 3 and 4 ). The coexistence of two phases extends over a broad pressure range and signals the sluggish character of the phase transition. A large pressure range of phase coexistence, of $10 \mathrm{GPa}$ or larger, has been observed for several firstorder structural phase transitions $\underline{16}-\underline{-18}$ and suggests that 


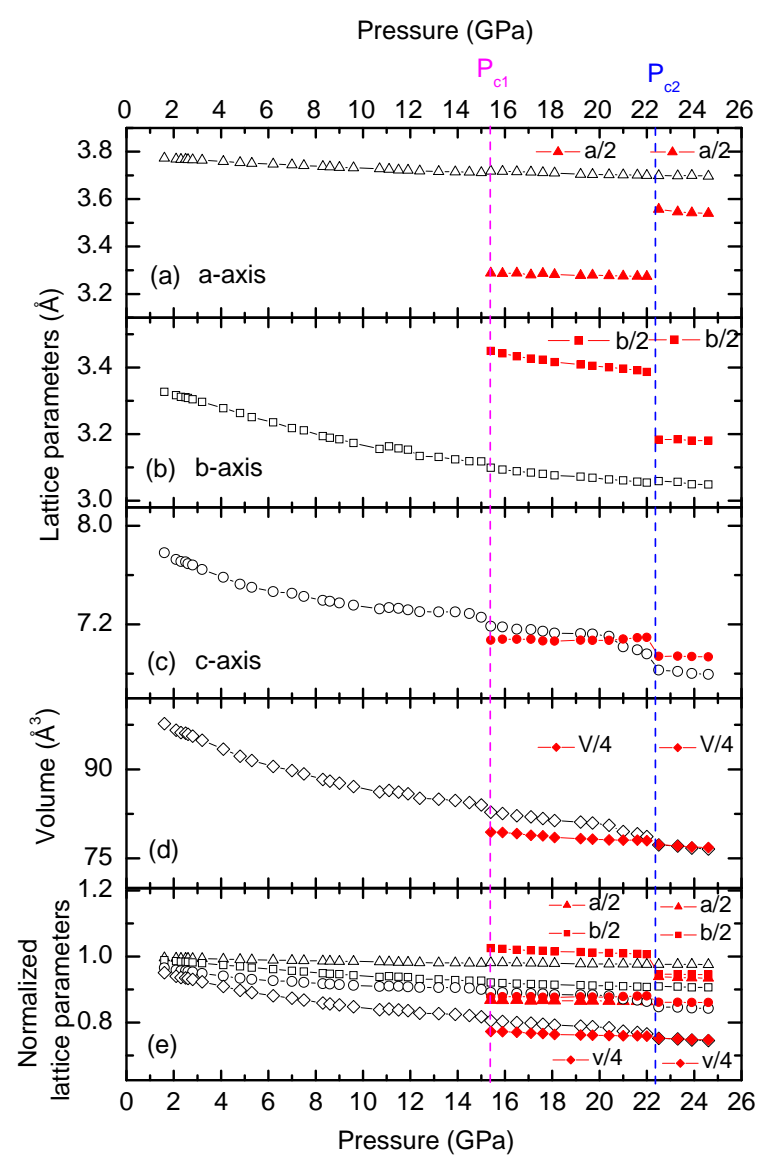

FIG. 3: (Color online) Results of the LeBail fits of the room-temperature $\mathrm{x}$-ray diffraction diagrams of $\mathrm{TiOCl}$ : lattice parameters and unit cell volume as a function of pressure [Pmmn: black, open symbols; $P 2_{1} / m$ : red (gray), full symbols]. Lines are guides to the eye. For the normalization of the lattice parameters and the unit cell volume the values of Ref 15 were used.

the two phases are almost energetically degenerate $\frac{19}{}$

It should be noted that already above $\sim 7 \mathrm{GPa}$ several weak reflections occur (marked with asterisks in Fig. 2 . which cannot be related to the orthorhombic phase $\mathrm{e}^{20}$ but to the monoclinic phase, which fully develops at $\mathrm{p}_{c 1}$ as described above. The appearance of diffraction peaks related to the monoclinic phase at pressures lower than $\mathrm{p}_{c 1}$ might be due to non-hydrostatic conditions in the DAC, leading to locally higher pressures than indicated by the ruby fluorescence.

The appearance of additional reflections above $\mathrm{p}_{c 1}$ due to a monoclinic phase in addition to those of the orthorhombic phase is also observed in the diffraction diagrams of Ref.5. There, the additional reflections were attributed to a monoclinic phase with a doubling of the unit cell along the $b$-axis. Furthermore, it was claimed ${ }^{5}$ that the monoclinic phase above $10 \mathrm{GPa}$ at room temperature resembles the monoclinic spin-Peierls phase occurring below the critical temperature $T_{S P}$ at ambient pressure. This led to the conclusion that $T_{S P}$ increases

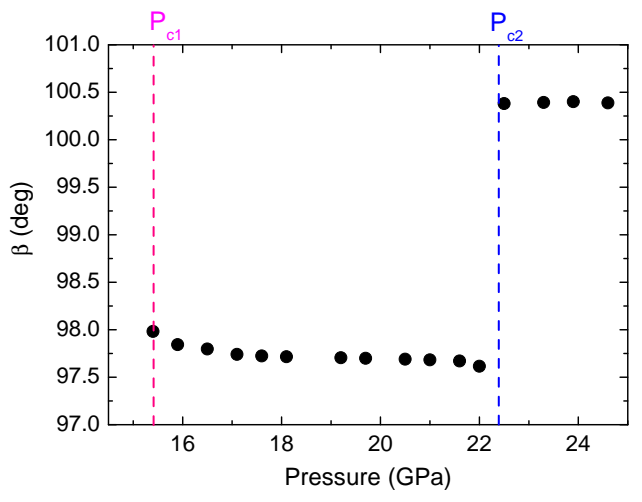

FIG. 4: Angle $\beta$ of the unit cell of the $P 2_{1} / m$ monoclinic crystal structure at room temperature as a function of pressure.

with increasing pressure, and it was proposed that $T_{S P}$ reaches room temperature for pressures above $10 \mathrm{GPa} . \underline{5}$ In contrast to the results of Blanco-Canosa et al. $\frac{5}{n}$ we find a doubling of the unit cell along both $a$ - and $b$-axes in the monoclinic cell. This discrepancy could be explained by the fact that the results of Ref $[5$ were restricted to the pressure range $\leq 15.2 \mathrm{GPa}$, where the monoclinic phase is not yet fully developed (which is the case only above $\mathrm{p}_{c 1}=15.4 \mathrm{GPa}$ according to our data). Furthermore, it is important to stress the differences between the crystal structures of the ambient-pressure spin-Peierls phase at low temperatures and the high-pressure dimerized phase at room-temperature: While the former shows an $a$-axis unique monoclinic symmetry with a monoclinic angle $\alpha \sim 90^{\circ}, 21-23$ the latter has a $b$-axis unique monoclinic symmetry with monoclinic angle $\beta \sim 99^{\circ}, \underline{5}$

It is interesting to note that an approximated doubling of the monoclinic unit cell along the $a$-axis was recently observed in a high-pressure x-ray diffraction study on Ti$\mathrm{OCl}$ at $T=6 \mathrm{~K}: \stackrel{9}{\underline{9}}$ Starting from the ambient-pressure, lowtemperature monoclinic spin-Peierls phase, Prodi et al. found a pressure-induced suppression of the dimerization along the $b$-axis in the vicinity of a first-order structural phase transition at around $13 \mathrm{GPa} . \stackrel{9}{*}$ The high-pressure phase shows an incommensurate superstructure of the type $(2 a-\epsilon) \times b \times c$ and might be interpreted in terms of a conventional Peierls state.

At $p_{c 2} \approx 22 \mathrm{GPa}$ again significant changes in the $\mathrm{x}$-ray diffraction diagram are observed (see Figs. 11and2), indicating the occurrence of a second structural phase transition: All lattice parameters of the monoclinic phase show pronounced anomalies at $p_{c 2}$, as seen in Figs. 3 and 4 . Above $p_{c 2}$ several weak reflections appear at diffraction angles below $10^{\circ}$, which can neither be related to the orthorhombic and monoclinic phase, nor explained in terms of reflections from the ruby chips, the diamond anvils or the gasket. In view of the complicated phase diagram of $\mathrm{TiOCl}$ under pressure, with a coexistence of multiple phases, we explain these weak reflections in terms of a third phase appearing above $p_{c 2}$. In order to specify the 


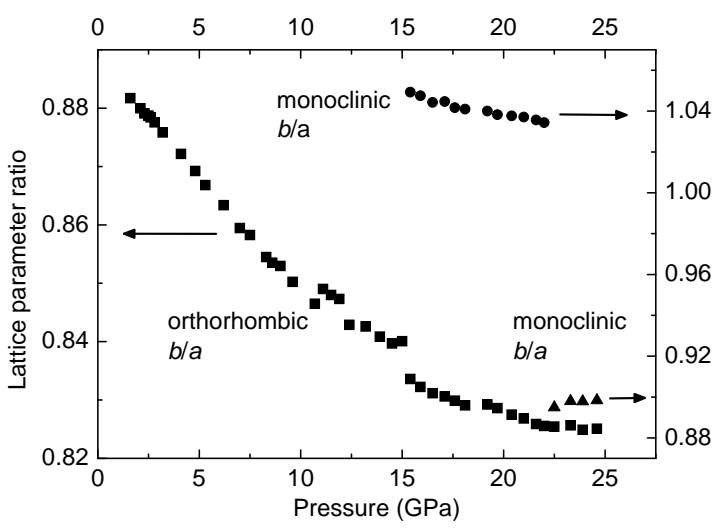

FIG. 5: Lattice parameter ratio as a function of pressure: Ratio $b / a$ for the orthorhombic phase (Pmmn, squares) for the whole studied pressure range; ratio $b / a$ for the monoclinic phase $\left(P 2_{1} / m\right.$, circles $)$ in the pressure range $p_{c 1}<p<p_{c 2}$; ratio $b / a$ for the monoclinic phase $\left(P 2_{1} / m\right.$, triangles $)$ in the pressure range $p>p_{c 2}$.

structural details of this third phase, x-ray diffraction data at higher pressures are needed.

In the following we speculate on the possible mechanism driving the structural phase transitions in $\mathrm{TiOCl}$ under pressure. We propose that their occurrence is closely linked to the tuning of the anisotropy or dimensionality of the system under pressure. For an illustration of the dimensionality, we plot and compare in Fig. 5 the lattice parameter ratios for the various phases. Here we assume that the average Ti-Ti distance scales with the lattice parameters. For the orthorhombic phase (Pmmn) the ratio $b / a$ is shown for the whole pressure range studied. For the high-pressure phases we take into account the superstructures and thus plot the ratio $b / a$ for the monoclinic phase $\left(P 2_{1} / m\right)$ in the pressure range $p>p_{c 1}$.

For the orthorhombic phase the ratio $b / a$ monotonically decreases with increasing pressure for the whole pressure range studied, with the tendency to saturation at high pressures. This indicates a pressure-induced enhancement of the one-dimensional character of the system consistent with an earlier report. $\underline{\underline{5}}$ The enhanced onedimensional character under pressure is due to the larger compressibility of the lattice along the $b$-direction. $\frac{\underline{5}}{\text { At }}$ $p_{c 1}$ a monoclinic phase with doubled unit cell along $a$ and $b$ is energetically degenerate with the orthorhombic phase. For this monoclinic phase we find a ratio $b / a$ close to one, i.e., the system is close to two-dimensional, up to $p_{c 2}$. This is due to the significant shrinkage of the unit cell along the $a$-direction and its enlargement along the $b$ direction, compared to the orthorhombic phase (Fig. 3). Accordingly, the interchain interaction is expected to play a major role in determining the electronic and magnetic properties of $\mathrm{TiOCl}$ in the monoclinic high-pressure phase. The importance of the interchain interaction in TiOCl at ambient pressure is commonly accepted $\underline{\underline{24}} \underline{\underline{26}}$ Our data suggest an enhanced interchain interaction, comparable to the intrachain interaction, for $p_{c 1}<p<p_{c 2}$.
Above $p_{c 2}$ the ratio $b / a$ drops to $\approx 0.9$, i.e., similar to the ratio $b / a$ of the orthorhombic phase at ambient conditions. Thus the material becomes more one-dimensional above $p_{c 2}$.

The occurrence of two pressure-induced phase transitions in $\mathrm{TiOCl}$ was predicted by Zhang et al. [6]: At $p_{c 1}$ a structural phase transition with a change from an orthorhombic (Pmmn) to a monoclinic crystal structure $\left(P 2_{1} / m\right)$ accompanied by a doubling of the unit cell along $b$ is expected. The concomitant insulator-to-metal transition is proposed to be due to a broadening of the electronic bands and a redistribution of electronic occupation among the three $t_{2 g}$ bands $\frac{6}{}$ A second first-order structural phase transition from the dimerized monoclinic $P 2_{1} / m$ phase to a uniform (undimerized) metallic phase with orthorhombic Pmmn symmetry is predicted at the critical pressure $p=p_{c 2} \approx 1.26 \cdot p_{c 1} \stackrel{6}{=}$ Because of the structural changes and the orbital repopulation a dimensional crossover of $\mathrm{TiOCl}$ from quasi-one-dimensional to quasi-two-dimensional was proposed to occur above $p_{c 2}: \underline{6}$ A tendency of the system at high pressures towards twodimensionality is consistent with our experimental results. However, it occurs already above $p_{c 1}$ and is limited to the pressure range $p_{c 1}<p<p_{c 2}$ according to our data. Discrepancies also exist regarding the doubling of the unit cell along the $a$ axis, and, furthermore, we do not find a pure undimerized, orthorhombic phase at high pressures, as theor $\mathrm{y}^{\underline{6}}$ predicts.

Interestingly, the pressure-induced structural instabilities seem to strongly depend on temperature: While at low temperature the dimerization along the $b$-axis becomes energetically unfavorable above $\sim 13 \mathrm{GPa}$ and a switching from the spin-Peierls phase to a phase with an incommensurate charge density wave along the $a$-axis occurs,$\frac{9}{}$ at room temperature the dimerization along $b$ for $p>p_{c 1}$ persists at least up to $25 \mathrm{GPa}$ according to our findings. Possible incommensurabilities of the superstructures at room temperature remain to be clarified.

\section{CONCLUSIONS}

Pressure-dependent x-ray powder diffraction data show the occurrence of two structural phase transitions in $\mathrm{TiOCl}$ at room temperature. Above $p_{c 1} \approx 15 \mathrm{GPa}$ a monoclinic phase (space group $P 2_{1} / m, b$-axis unique) with a dimerization along the $a$ - and $b$-direction emerges. At $p_{c 2} \approx 22 \mathrm{GPa}$ all lattice parameters of the monoclinic phase show pronounced anomalies. A fraction of the sample persists in the ambient orthorhombic phase (space group Pmmn) over the whole pressure range studied.

\section{Acknowledgements}

We acknowledge the ESRF Grenoble for the provision of beamtime. Financial support by the DFG, including 
the Emmy Noether-program, SFB 484, DFG-CL124/6-1, and Sm55/15, is acknowledged.

* Email: christine.kuntscher@physik.uni-augsburg.de

1 C. A. Kuntscher, S. Frank, A. Pashkin, M. Hoinkis, M. Klemm, M. Sing, S. Horn, and R. Claessen, Phys. Rev. B 74, 184402 (2006).

2 C. A. Kuntscher, S. Frank, A. Pashkin, H. Hoffmann, A. Schönleber, S. van Smaalen, M. Hanfland, S. Glawion, M. Klemm, M. Sing, S. Horn, and R. Claessen, Phys. Rev. B 76, 241101(R) (2007).

3 C. A. Kuntscher, M. Klemm, S. Horn, M. Sing, and R. Claessen, Eur. Phys. J. Special Topics 180, 29 (2010).

${ }^{4}$ M. K. Forthaus, T. Taetz, A. Möller, and M. M. AbdElmeguid, Phys. Rev. B 77, 165121 (2008).

5 S. Blanco-Canosa, F. Rivadulla, A. Pineira, V. Pardo, D. Baldomir, D. I. Khomskii, M. M. Abd-Elmeguid, M. A. Lopez-Qintela, and J. Rivas, Phys. Rev. Lett. 102, 056406 (2009).

6 Y.-Z. Zhang, H. O. Jeschke, and R. Valenti, Phys. Rev. Lett. 101, 136406 (2008).

7 C. A. Kuntscher, A. Pashkin, H. Hoffmann, S. Frank, M. Klemm, S. Horn, A. Schönleber, S. van Smaalen, M. Hanfland, S. Glawion, M. Sing, and R. Claessen, Phys. Rev. B 78, 035106 (2008).

${ }^{8}$ C. A. Kuntscher, J. Ebad-Allah, A. Pashkin, S. Frank, M. Klemm, S. Horn, A. Schönleber, S. van Smaalen, M. Hanfland, S. Glawion, M. Sing, and R. Claessen, High Pressure Res. 29, 509 (2009).

9 A. Prodi, J. S. Helton, Y. Feng, and Y. S. Lee, arXiv: 0908.0518 (2009).

${ }^{10}$ H. Schäfer, F. Wartenpfuhl, and E. Weise, Z. Anorg. Allg. Chem. 295, 268 (1958).

11 H. G. von Schnering, M. Collin, and M. Hassheider, Z. Anorg. Allg. Chem. 387, 137 (1972).

12 H. K. Mao, J. Xu, and P. M. Bell, J. Geophys. Res. 91, 4673 (1986).

13 A. Hammersley, computer program FIT2D (ESRF, Grenoble, 1998).

14 V. Petricek, M. Dusek, and L. Palatinus. Jana2006. A crystallographic computing system. Institute of Physics, Praha, Czech Republic (2006).

15 T. Sasaki, M. Mizumaki, K. Kato, Y. Watabe, Y. Mishihata, M. Takata, and J. Akimitsu, J. Phys. Soc. Jpn. 74, 2185 (2005).

16 G. Kh. Rozenberg, M. P. Pasternak, P. Gorodetsky, W. M. Xu, L. S. Dubrovinsky, T. Le Bihan, R. D. Taylor, Phys. Rev. B 79, 214105 (2009).

17 I. Loa, K. Syassen, X. Wang, F. Lichtenberg, M. Hanfland, and C. A. Kuntscher, Phys. Rev. B 69, 224105 (2004).

18 W. M. Xu, O. Naaman, G. Kh. Rozenberg, M. P. Pasternak, and R. D. Taylor, Phys. Rev. B 64, 094411 (2001).

19 Please note that the volume fractions of the phases cannot be extracted based on the LeBail fits.

20 The appearance of reflections above a certain pressure can be related to the loss of the preferred orientation of the crystallites in the DAC, as for example observed in the $12.7 \mathrm{GPa}$ diffraction diagram, where a new reflection related to the orthorhombic phase is observed between $2 \theta=14^{\circ}$ and $15^{\circ}$.

21 M. Shaz, S. van Smaalen, L. Palatinus, M. Hoinkis, M. Klemm, S. Horn, and R. Claessen, Phys. Rev. B 71, 100405(R) (2005).

22 A. Schönleber, S. van Smaalen, and L. Palatinus, Phys. Rev. B 73, 214410 (2006).

23 A. Schönleber, G. Shcheka, and S. van Smaalen, Phys. Rev. B 77, 094117 (2008).

24 D. Fausti, T. T. A. Lummen, C. Angelescu, R. Macovez, J. Luzon, R. Broer, P. Rudolf, P. H. M. van Loosdrecht, N. Tristan, B. Büchner, S. van Smaalen, A. Möller, G. Meyer, and T. Taetz, Phys. Rev. B 75, 245114 (2007).

${ }^{25}$ R. Rückamp, J. Baier, M. Kriener, M. W. Haverkort, T. Lorenz, G. S. Uhrig, L. Jongen, A. Möller, G. Meyer, and M. Grüninger, Phys. Rev. Lett. 95, 097203 (2005).

26 Y.-Z. Zhang, H. O. Jeschke, and R. Valenti, Phys. Rev. B 78, 205104 (2008). 